\title{
One-Month versus Three-Month Formulation of Paliperidone Palmitate Treatment in Psychotic Disorders: Patients', Relatives', and Mental Health Professionals' Perspectives
}

\author{
S Kor Spoelstra ${ }^{1,2}$, Jojanneke Bruins ${ }^{2}$, Leonie Bais ${ }^{2}$, Paul Seerden ${ }^{3}$, Stynke Castelein ${ }^{2,4}$, \\ Henderikus Knegtering ${ }^{2,5}$
}

'Addiction Care North Netherlands, Groningen, the Netherlands; ${ }^{2}$ Lentis Psychiatric Institute, Groningen, the Netherlands; ${ }^{3} \mathrm{GGZ}$ Friesland Psychiatric Institute, Leeuwarden, the Netherlands; ${ }^{4}$ University of Groningen, Faculty of Behavioural and Social Sciences, Department of Clinical Psychology and Experimental Psychopathology, Groningen, the Netherlands; ${ }^{5}$ University Medical Center Groningen, University of Groningen, Rob Giel Research Center, Groningen, the Netherlands

Correspondence: S Kor Spoelstra, Addiction care North Netherlands, Leonard Springerlaan 27, Groningen, 9727 KB, the Netherlands, Tel +3I503648900, Fax +3I503648999, Email k.spoelstra@vnn.nl

\begin{abstract}
Purpose: Paliperidone palmitate is the only available long acting injectable (LAI) antipsychotic with a monthly and three-monthly formulation. LAIs may help battle non-adherence. Studies about the experiences of switching from the monthly (PP1M) to the threemonthly formulation (PP3M) of paliperidone are scarce. Therefore, the aim of this study is to evaluate the perspectives of patients, relatives, and mental health professionals on PP3M compared with PP1M.
\end{abstract}

Material and Methods: This was a multicenter, retrospective, non-interventional one-time questionnaire survey among patients with psychotic disorders who switched from PP1M to PP3M $(n=38)$, their relatives $(n=13)$ and mental health professionals $(n=38)$. General satisfaction and (un)desired effects were measured using the Medication Satisfaction Questionnaire (MSQ) and the Subjects' Reaction to Antipsychotics (SRA), respectively. Additional questionnaires assessed socio-demographic variables, preference, effectiveness, side-effects, and confidence in PP3M compared to PP1M.

Results: Mean number of received PP3M injections was 4.2 (SD 2.5). The three study groups reported a high level of confidence in PP3M. High general satisfaction rates about PP3M among patients $(69 \%)$ and mental health professionals $(95 \%)$ were reported. The majority of patients, relatives, and mental health professionals reported similar or in some cases even greater effectiveness and similar or in some cases even less side-effects of PP3M compared to PP1M. Sixty-seven percent of the relatives reported less concerns about non-adherence after switching to PP3M.

Conclusion: Most patients, relatives, and mental health professionals prefer PP3M over PP1M. The positive attitudes of all parties may facilitate the more frequent use of PP3M and potentially the clinical outcomes.

Keywords: paliperidone palmitate 3-monthly, long-acting injectable antipsychotics, psychotic disorders, mental health professionals, family, perspectives

\section{Introduction}

Both in general medicine and psychiatry, medication adherence is a significant challenge for patients, relatives and clinicians. ${ }^{1}$ Non-adherence to treatment with oral antipsychotic medication, e.g., due to a lack of effectiveness or undesired side effects, is common among people with severe psychotic symptoms, ${ }^{2}$ with self-reported percentages ranging from $30 \%$ to $50 \% .^{3,4}$ Negative consequences of non-adherence are relapses, impaired functioning, lower quality of life, suicidality, premature mortality, and increased costs for the healthcare system. ${ }^{5}$ Targeting or circumventing nonadherence is therefore of great importance in the treatment of psychotic disorders. ${ }^{6}$ 
One widely used strategy to circumvent non-adherence to antipsychotic medication and to subsequently improve clinical outcomes is treatment with long-acting injectable (LAI) antipsychotic formulations. LAIs are mostly administered with biweekly to four-weekly intervals (depending on the type of drug) and contribute to the successful maintenance treatment of psychotic disorders. ${ }^{7,8}$ Emerging evidence suggests that LAIs are favorable compared to oral antipsychotic drugs. ${ }^{8}$ Among others, the risk of rehospitalization is about $20 \%$ to $30 \%$ lower in LAI treatment compared to most equivalent oral formulations. ${ }^{9}$

In theory, LAIs offer several biological advantages over oral formulations. They avoid first-pass metabolism, have a greater bioavailability and guarantee a more stable plasma concentration. ${ }^{10}$ When dosing is done carefully, the risk of an overdose or an intoxication is lower with LAIs compared to oral formulations. The longer time intervals between injections and a more stable daily functioning provide more time for the treatment of other problems related to the disorder, such as substance abuse, trauma and social recovery. From a cognitive perspective, LAIs have the advantage that patients do not have to remember to take their daily medication. An additional benefit may be that LAIs are administered during visits to a mental health professional, which makes it easy to know when patients stop their treatment and become at risk for a relapse. ${ }^{6}$ Disadvantages of LAIs include dosage inflexibility and the relatively long duration for medication effects to wear off in case of side-effects. ${ }^{6}$

The lower percentage of relapse and longer timespan to relapse with LAI antipsychotic formulations suggest that longer-acting may be preferred over shorter acting formulations, especially in patients with adherence issues. ${ }^{7}$ LAIs with a duration longer than 1 month might have additional benefits for patients to current existing LAIs. Long(er) dosage intervals mean less frequent injections and subsequently less pain or fear for these injections, less dependence on contacts with clinicians and a decreased necessity to travel for receiving an injection.

Currently, a paliperidone palmitate version with therapeutic blood-levels for 3-months (PP3M) is the only available LAI antipsychotic that offers an extended 3-month window of stable plasma drug concentration, meaning patients only need four injections per year. ${ }^{7}$ PP3M is available for the treatment of adult patients with schizophrenia who previously received adequate treatment with the one-monthly formulation (PP1M) at least four times. ${ }^{11}$ PP3M, which received FDA approval in 2015, has demonstrated favourable symptomatic- and functional outcomes. ${ }^{12}$ To push the interval boundaries even further, a 6-month version of paliperidone palmitate (PP6M) is currently under investigation.

Shared decision-making increases adherence in people with schizophrenia, as does collaboration among mental health professionals, patients and relatives (ie, parents, siblings, neighbors or friends). ${ }^{13}$ It could also enhance insight into the psychotic disorder and lead to better treatment expectations and outcomes. ${ }^{14,15}$ Their perspectives are vital in the discussion surrounding LAIs and their embedding in overall treatment strategies. However, research on experiences of mental health care professionals, patients and relatives with PP3M is scarce.

A study of Cassidy and Miles (2020) evaluated by an online-questionnaire the impact of PP3M on patient management, including non-adherence and relapse, from a psychiatrist $(n=7)$ and nurse $(n=17)$ perspective. ${ }^{16}$ Treatment goals were met or exceeded, and psychiatrists were satisfied with treatment efficacy and relapse prevention. They concluded that from a clinician's perspective, PP3M offers patients the potential to remain adherent and improve social functioning. An online questionnaire survey of Pai et al among 16 prescribers concluded that they had the impression that patients were satisfied with symptom control and general functioning with PP3M. ${ }^{17}$ From a patient's perspective, individual depth-interviews with 24 patients with schizophrenia revealed that they had more feelings of stability and were more physically and socially active after they received PP3M. ${ }^{18}$ The authors concluded that switching from PP1M to PP3M seemed to be experienced as advantageous for patients with psychotic disorders.

The aim of the present study is to evaluate the perspectives of patients, their relatives and mental health professionals on the (dis)advantages of PP3M compared to PP1M.

\section{Materials and Methods}

\section{Subjects}

\section{Patients}

Patients were eligible for inclusion when they 1 ) were diagnosed with a psychotic disorder in accordance with the DSM-5; ${ }^{19}$ ) received voluntarily treatment with PP1M for at least 4 months before voluntarily switching to PP3M monotherapy; 3 ) had 
started using PP3M about 1 year ago, of which they had at least one injection; and 4) were willing to participate and able to give oral and written consent for participation.

\section{Relatives}

Relatives were included when they 1) were identified by patients as being an important relative; 2) got permission from patients to participate in the study; and 3) were able to give oral and written informed consent.

\section{Mental Health Professionals}

Mental health professionals were eligible for inclusion when they 1) treated patients with psychotic disorders; 2) treated patients who had used PP1M for at least 4 months followed by a switch to PP3M, and 3) were able to give oral and written informed consent.

\section{Procedure}

Mental health professionals from five mental health institutions in the Northern Netherlands were contacted about this study through training networks, conferences or by the researchers personally. Willing professionals were approached by a research nurse (BK, AZ, KH) and received study details per e-mail. Professionals were asked to fill out questionnaires about all patients in their caseload who met the inclusion criteria and were subsequently asked to contact these eligible patients for participation in this study. Participating patients received oral and written information about the study, a consent form and the questionnaires from their mental health professional. Patients who had consented to participate were asked for permission to contact an important relative, in order to get their perspective on PP3M as well. After patients' consent, relatives also received information and questionnaires from the mental health professional. The mental health professionals were provided with a return envelope to mail the completed consent forms and questionnaires back to the researchers. Data was collected from March 2018 to January 2020. If needed, the research nurses also sent reminders and provided additional assistance to the mental health professionals.

The study is performed in accordance with the Good Clinical Practice Guidelines, The Declaration of Helsinki and Dutch legislation on human research and privacy. As this non-interventional study would cause minimal burden to any party, the Medical Ethical Committee of the University Medical Center Groningen judged that the study was not subject to the Dutch Medical Research Involving Human Subjects Act (WMO).

\section{Measures}

Patients

Patients filled in a questionnaire about their experiences with PP3M (effectiveness, side-effects and confidence, and preference) compared to PP1M. They also filled in the Medication Satisfaction Questionnaire (MSQ), which measured their satisfaction with PP3M treatment. The MSQ is a single-item self-report questionnaire, where satisfaction is rated on a 7-point Likert scale (1=extremely dissatisfied; 7=extremely satisfied), and has adequate psychometric properties for people with psychotic disorders. ${ }^{20}$ Patients also filled in the adapted Subjects' Reaction to Antipsychotics about PP3M (SRA-34), ${ }^{21}$ a 34-item self-report questionnaire where patients rated each potential desired $(n=10)$ and undesired $(n=24)$ effect on a 3-point Likert scale ( $1=$ not present, $2=$ yes, somewhat present and $3=y e s$, strongly present). Both subscales have been shown to have a good internal consistency (Cronbach's alpha $=0.82$ ). In this study, $\alpha=0.90$ for the desired and $\alpha=0.82$ for the undesired subscale.

Additionally, patients were asked to provide demographic information about themselves (age, gender), but also about duration of (long-acting) antipsychotic use, duration of PP3M use, number of PP3M injections, motivation to switch from PP1M to PP3M, and change of number of contacts with mental health professionals.

\section{Relatives}

Relatives filled in a questionnaire about effectiveness, side-effects, confidence, and relatives' burden in PP3M compared to PP1M. Furthermore, relatives were asked to disclose their age, gender, relation to the patient, their means of contact (eg, by phone, WhatsApp, e-mail or in person) and the average frequency of their contact with the patient per week or per month. 


\section{Mental Health Professionals}

Mental health professionals filled in a questionnaire about their experiences with PP3M (effectiveness, side-effects and confidence, and preference) compared to PP1M. They also filled in the MSQ to indicate their satisfaction with PP3M. In addition, mental health professionals were asked to provide demographic information about themselves (age, gender, profession, length of clinical experience in present profession, and workplace; in or outpatient setting), as well about their participating patients (age, gender, classification, duration of psychotic disorder, realization of the necessary to use antipsychotics, duration of (long-acting) antipsychotics use, duration of PP3M use, number of PP3M injections, motivation to switch from PP1M to PP3M and change of number of contacts with a mental health professional).

\section{Data Analysis}

Descriptive analyses (ie, frequencies, means and standard deviations) were used to describe the demographic characteristics and evaluate the outcomes of the questionnaires. All data were analyzed using IBM SPSS 25.0. ${ }^{22}$

\section{Results}

\section{Response}

In total, 44 couples of patients and mental health professionals were included in this study. In 32 of these 44 couples, both the patient and the treating mental health professional answered the questions. Of the remaining 12 couples there were six couples where we only received a response from the professionals, but not from the patient. Similarly, of the last six couples we only received a response from the patients, but not from their mental health professional. The majority of the included patients (53\%) had received at least four injections with PP3M at the time of assessment. Furthermore, we included 13 relatives of participating patients, most of which were parents (54\%). An overview of the sample characteristics is provided in Table 1 .

\section{Patients}

Patients were somewhat satisfied (21\%), very satisfied (61\%) or extremely satisfied (8\%) with using PP3M (see Table 2). Two patients (5\%) were neutral and the two patients $(5 \%)$ who were unsatisfied with PP3M gave as primary reason that they did not want to use any (LAI) medication at all. The majority of the patients evaluated PP3M as equally effective $(84 \%)$ or better (10\%) than PP1M (see Table 2). Desired effects of PP3M most reported by patients on the SRA-34 were feeling more cheerful (61\%), sleeping better (58\%), having more control over their thoughts (53\%), more attention for their environment (45\%), being able to think more clearly (45\%) and interacting easier with others $(42 \%)$.

The majority of the patients also reported that PP3M caused equal $(58 \%)$ or less $(21 \%)$ side effects than PP1M. Undesired side effects of PP3M most reported by patients on the SRA-34 were weight gain (47\%), blunted affect (40\%), rigid muscles (34\%), physical tiredness (34\%) increased appetite (32\%) and trouble waking up (32\%). When asked about any differences in side effects between PP3M and PP1M, 23\% of the patients reported fewer side effects from PP3M (ie, less problems with body cramping $(n=1)$, hunger $(n=1)$, sweating $(n=1)$, shaking $(n=1)$, stomach $(n=1)$, sleeping $(n=1)$ and tiredness $(\mathrm{n}=2)$ ) against $12 \%$ who reported more side effects from PP3M (ie, more problems with shaking $(\mathrm{n}=1)$, dizziness $(\mathrm{n}=1)$, fatigue $(\mathrm{n}=1)$, blunted affect $(\mathrm{n}=1)$ and having colds $(\mathrm{n}=1))$.

When asked about their motivation to switch from PP1M to PP3M, patients named fewer confrontations with the necessity to use medication (42\%), fewer painful injection moments (32\%), less need for travelling to appointments (24\%), fewer risk of missing an appointment (18\%) and less moments of experiencing shame (8\%) as their main reasons.

Two of the patients (5\%) preferred PP1M over PP3M, whereas $82 \%$ preferred to use PP3M (see Table 2). One patient switched back to PP1M, because of the experienced side effects (type unknown), but all others (97\%) were still using PP3M at the time of the assessment. 
Table I Sample Characteristics

\begin{tabular}{|c|c|c|c|}
\hline & Patients $(n=38)$ & Relatives $(n=13)$ & Mental Health Professionals $(n=38)$ \\
\hline Age in years, $M(S D)$ & $45.6(10.2)$ & $50.7(14.0)$ & $48.0(7.3)$ \\
\hline Male gender, \% & 61 & 31 & 66 \\
\hline \multicolumn{4}{|l|}{ Profession, \% } \\
\hline Psychiatrist & - & & 47 \\
\hline Nurse practitioner & - & & 24 \\
\hline Social psychiatric nurse & - & & 13 \\
\hline Case manager & - & & 10 \\
\hline Psychologist & - & & 5 \\
\hline Treatment experience (years), M (SD) & & & $16.7(8.2)$ \\
\hline \multicolumn{4}{|l|}{ Diagnosis, \% } \\
\hline Schizophrenia & 79 & & \\
\hline Other & 21 & & \\
\hline \multicolumn{4}{|l|}{ Duration of antipsychotic drug use, $\%$} \\
\hline$<5$ years & 32 & & \\
\hline $5-10$ years & 5 & & \\
\hline 10 years & 63 & & \\
\hline \multicolumn{4}{|l|}{ Duration of PPIM use, \% } \\
\hline 4-12 months & 55 & & \\
\hline$>12$ months & 45 & & \\
\hline Number of PP3M injections, $M(S D)$ & $4.15(2.5)$ & & \\
\hline \multicolumn{4}{|l|}{ Relation to the patient, \% } \\
\hline Parent & & 54 & \\
\hline Partner & & 15 & \\
\hline Neighbor & & 8 & \\
\hline Other & & 23 & \\
\hline
\end{tabular}

\section{Relatives}

Relatives evaluated the effectiveness of PP3M as better than $(15 \%)$ or the same $(85 \%)$ as PP1M, and thought PP3M had equal $(69 \%)$ or less $(23 \%)$ side effects than PP1M (see Table 2).

All but one of the relatives had face-to-face contact with the patient. The majority (54\%) had in person contact on a daily basis. The in-person contact frequency of the remaining $46 \%$ varied from multiple times a week to one or twice per month. When asked about their concern with regard to the patient taking his/her medication, $67 \%$ reported that they were less worried since the patient started using PP3M, 17\% had no opinion and $16 \%$ was either more or equally worried.

In total, $85 \%$ of the relatives had confidence in PP3M and 77\% preferred PP3M over PP1M, whereas the remaining $23 \%$ had no preference (see Table 2). 
Table 2 Evaluation of PP3M, Separated by Participants Groups

\begin{tabular}{|c|c|c|c|}
\hline & Patients $(n=38)$ & Relatives $(n=13)$ & Mental Health Professionals $(n=38)$ \\
\hline & $\%(n)$ & $\%(n)$ & $\%(n)$ \\
\hline \multicolumn{4}{|l|}{ General satisfaction with PP3M } \\
\hline Extremely satisfied & $8(3)$ & & $8(3)$ \\
\hline Very satisfied & $61(23)$ & & $87(33)$ \\
\hline Somewhat satisfied & $21(8)$ & & $5(2)$ \\
\hline Not satisfied, not unsatisfied & $5(2)$ & & 0 \\
\hline Somewhat unsatisfied & 0 & & 0 \\
\hline Very unsatisfied & 0 & & 0 \\
\hline Extremely unsatisfied & $5(2)$ & & 0 \\
\hline \multicolumn{4}{|c|}{ Experienced effectiveness of PP3M vs PPIM } \\
\hline PP3M > PPIM & $10(4)$ & $15(2)$ & $26(10)$ \\
\hline PP3M = PPIM & $84(32)$ & $85(2)$ & $71(27)$ \\
\hline PP3M $<$ PPIM & $3(1)$ & 0 & $3(1)$ \\
\hline Neither works & $3(1)$ & 0 & 0 \\
\hline \multicolumn{4}{|c|}{ Experienced side-effects of PP3M vs PPIM } \\
\hline PP3M > PPIM & $10(4)$ & $8(1)$ & 0 \\
\hline$P P 3 M=P P I M$ & $58(22)$ & $69(9)$ & $66(25)$ \\
\hline PP3M $<$ PPIM & $21(8)$ & $23(3)$ & $24(9)$ \\
\hline No opinion & $10(4)$ & 0 & $10(4)$ \\
\hline \multicolumn{4}{|l|}{ Confidence in PP3M } \\
\hline Yes & $90(34)$ & $85(15)$ & $97(37)$ \\
\hline No & $10(4)$ & $15(2)$ & $3(1)$ \\
\hline \multicolumn{4}{|l|}{ Preference } \\
\hline PP3M & $82(3 I)$ & $77(10)$ & $97(37)$ \\
\hline PPIM & $5(2)$ & 0 & 0 \\
\hline No preference & $13(5)$ & $23(3)$ & $3(1)$ \\
\hline
\end{tabular}

\section{Mental Health Professionals}

The majority of mental health professionals (95\%) was satisfied with PP3M. The only limitation they mentioned was that it is not possible to quickly change the dosage of PP3M. The majority of the mental health professionals evaluated PP3M as equally effective $(71 \%)$ or better $(26 \%)$ and with equal $(66 \%)$ or less side effects $(24 \%)$ than PP1M (see Table 2$)$. When asked to elaborate on the side-effects, they mentioned a decrease in appetite $(n=1)$ and tiredness $(n=1)$ in patients. Of the following side-effects they were uncertain whether these were more or less severe in comparison with PP1M: weight gain $(n=2)$, sedation $(n=3)$, blunted affect $(n=2)$ and tremors $(n=1)$. The mental health professionals who listed these side effects did report that PP3M had fewer side effects than PP1M in general. 
Furthermore, most mental health professionals reported that the number of contact moments with their patients had either stayed the same (53\%) or decreased (42\%). All but one of the professionals (97\%) had confidence in PP3M and preferred it above PP1M, with the one professional having no preference (see Table 2).

\section{Discussion}

The collective experience of patients, relatives, and mental health professionals was that PP3M works equally well or in some cases even better and had equal or in some cases even less side effects than PP1M. The high satisfaction rate of patients $(69 \%)$ in our study is in line with a study of Fernández-Miranda that showed more satisfaction with PP3M compared to PP1M. ${ }^{23}$ The majority of patients, relatives, and mental health-care professionals preferred PP3M over PP1M, but partially for different reasons. Patients reported less confrontation with the necessity to use medication, fewer painful injections, less travelling and less moments of experiencing shame. Their relatives, mostly parents, had less concerns about non-adherence, a diminished feeling of obligation and therefore less resistance, and reported less disruption of daily life partly due to less outpatient clinic visits. Mental health-care professionals named less concerns about adherence, less stigma and fewer confrontations for their patients with the need for (painful) injections.

The finding of considerably less burden for relatives (67\%) is in accordance with a study of Garcia-Portilla (2020), and supports the hypothesis that PP3M can alleviate relatives' burden. ${ }^{12}$ In our study, 7 of 13 relatives (54\%) had daily contact with the patient, while $85.7 \%$ out of them experienced less concerns about medication intake.

Previous studies found either that the transition from PP1M to PP3M was associated with a decline in outpatient and pharmacy visits ${ }^{24}$ or that contact frequency was not influenced by PP3M. ${ }^{18}$ Our results suggest that this may be individually determined, essentially supporting both findings, with approximately half of our participants reporting an unchanged number of contact moments (53\%) and the other half reporting a reduced number of contact moments $(42 \%)$. Although our participants did not specify the reasons for any of their visits, it is likely that at least the visits exclusively intended for PP3M injections (four times a year) were less frequent compared to PP1M (twelve times a year). A reduction in mental health associated contacts could hypothetically be associated with hope and social acceptability, which would likely be appreciated by patients and their relatives. ${ }^{14}$ It is possible that pharmacological contacts were replaced with non-pharmacological intervention contacts in patients with an unchanged number of visits during their use of PP3M.

Previous literature studies suggest that LAIs are sometimes considered as coercive, old-fashioned and more expensive than oral medication, both by patients and mental health professionals. ${ }^{25,26}$ Our findings do not support this, with none of the participants reporting a sense of coerciveness or increased financial burden. This suggests that LAIs can also be considered as a modern and comfortable treatment option for patients. Although PP3M is more expensive than oral or other depot antipsychotics, it is fully reimbursed by the Dutch healthcare system, making the financial argument obsolete. The only limitation of PP3M reported in this study was by mental health professionals: that it is not possible to change the dosage of PP3M immediately. The mostly positive attitudes, expressed as preference, of all involved parties towards the switch from PP1M to PP3M may play an important role in facilitating the use of PP3M, since existing negative attitudes of patients and mental health professionals towards LAIs are currently an important reason why they are underutilized. ${ }^{27}$

The experiences of patients, relatives, as well mental health professionals support a recovery-oriented approach, which encourages collaboration. Members of the patient's support network play a critical role in the treatment plan (e.g., assisting the patient in decision-making) and recovery process. ${ }^{28}$ Providing significant others with psycho-education about the potential risks, benefits and experiences of others with LAIs could support them in this role. From a recovery point of view: the use of LAIs antipsychotics could not just be considered a tool for preventing relapse but also a resource to help patients work towards their own recovery goals. ${ }^{29}$ Not only symptomatic remission is important in this context. Functional remission might be even more important considering its association with real-world outcomes, such as social and occupational functioning and living independently. ${ }^{12}$ 


\section{Strength and Limitations}

To our knowledge, the present study is the first to evaluate satisfaction and confidence about PP3M, in the triad of patients, relatives, and mental health professionals. Especially, the reported experiences of the generally not included relatives is a major strength of this study.

The study limitations include a relatively small sample size and the retrospective design, which could have resulted in recall bias. Especially recruiting relatives in the study turned out to be very difficult. However, compared to previous published studies, our study has a high sample size. ${ }^{16-18}$

Selection bias may also have influenced the results, since LAIs antipsychotics are generally underutilized and are most often prescribed by mental health professionals who already have a positive attitude towards LAI antipsychotics. ${ }^{30}$ Nevertheless, the findings of our study are in accordance with the results of Cassidy and Miles, who found high satisfaction with PP3M in psychiatrists and nurses. Moreover, patients in our study had already received years of antipsychotic treatment $(63 \%>10$ years), which makes it difficult to generalize our findings to other populations (e.g., first-episode psychosis). Furthermore, our participants had a relatively high age, and it is unknown whether perspectives are age-, experience- or cultural dependent. We were neither able to measure whether there was indeed time to focus on other aspects of treatment and recovery nor the cost-effectiveness of PP3M.

\section{Future Research}

We recommend future studies to include a prospective design and evaluate experiences of stakeholders with (side) effects and treatment outcomes of PP3M, such as social and cognitive functioning, stigmatization, therapeutic alliance and recovery of patients. Monitoring contacts with mental health practitioners should preferable be monitored with validated questionnaires. Levels of stigmatization are an important outcome to monitor as well. Although LAIs could also be associated with more stigma because of the belief that LAIs reflect a more severe psychotic disorder, ${ }^{26,31}$ our findings suggest that less frequent confrontation with medication may lead to less experienced stigma, potentially because of a feeling of recovery. This is in line with a study of Pungor et al that showed a decreased perceived stigma after the switch from PP1M to PP3M. ${ }^{32}$ It is also worth evaluating whether the prolonged dosage interval of PP3M may put focus on other aspects of treatment (e.g., substance abuse, trauma, physical condition and social recovery) to achieve treatment goals. ${ }^{33}$ The long dosage interval of PP3M may influence mental health care organization with regard to policies, infrastructure (e.g., storage of medication) and procedures. Evaluating cost-effectiveness might be able to demonstrate that PP3M reduces total costs due to increased adherence and subsequently less relapses and admissions. LAIs are generally more expensive compared to oral formulations. ${ }^{34-36}$ In contrast, overall cost-effectiveness studies demonstrated that PP3M may reduce total costs, a finding that asks for confirmation in non-industry sponsored studies. ${ }^{37}$ Future studies may consider to include a standardized care consumption questionnaire such as the TIC-P. ${ }^{38}$

A prospective study design including relatives from the beginning of treatment may teach us more on how to provide assistance in the complex dynamics of relatives and patients with psychotic disorders.

\section{Conclusion}

Patients, their relatives and mental health professionals reported a high satisfaction rate and high levels of confidence with PP3M, with similar or greater effectiveness and similar or less side-effects of PP3M compared to PP1M. The majority preferred PP3M over PP1M. Further prospective studies are needed to examine effectiveness, side effects and perspectives of PP3M and other LAIs in more detail, to see if they influence clinical practice and treatment outcome.

\section{Acknowledgments}

We gratefully acknowledge Bernadine Kralt, Anneke Zijlstra, and Kas Haneveer (research assistance) for data collection. 


\section{Funding}

Financial support was obtained from Janssen-Cilag BV, the Netherlands, which has marketing authorization for PP1M and PP3M in Europe. Janssen-Cilag BV was neither involved in the design and conduction of the study, nor in the interpretation of the results. Financial support was used to enable the appointment of a research assistant to recruit participants and collect data.

\section{Disclosure}

HK is a consultant and on the speakers list for Lilly, Lundbeck, Janssen-Cilag BV and Sunovion. PS is on the speakers list for Janssen-Cilag BV. Psychologists JB and LB, sociologist SC, and psychiatrist SKS are not on any speakers list of a pharmaceutical company nor being paid by Janssen-Cilag BV. The authors report no other conflicts of interest in this work.

\section{References}

1. Zullig LL, Ramos K, Bosworth HB. Improving medication adherence in coronary heart disease. Curr Cardiol Rep. 2017;19(11):113. doi:10.1007/ s11886-017-0918-y

2. Velligan DI, Sajatovic M, Hatch A, Kramata P, Docherty JP. Why do psychiatric patients stop antipsychotic medication? A systematic review of reasons for nonadherence to medication in patients with serious mental illness. Patient Prefer Adherence. 2017;11:449-468. doi:10.2147/PPA. S 124658

3. McIntosh AM, Conlon L, Lawrie SM, Stanfield AC. Compliance therapy for schizophrenia. Cochrane Database Syst Rev. 2006 ;(3):CD003442. doi:10.1002/14651858.CD003442.pub2

4. Marshall M, Rathbone J. Depot early intervention for psychosis. Cochrane Database Syst Rev. 2011;(6):CD004718. doi:10.1002/14651858. CD004718.pub3

5. Caripiniello B, Pinna F. Critical appraisal of 3-monthly paliperidone depot injections in the treatment of schizophrenia. Drug Des Devel Ther. 2016;24:1731-1742. doi:10.2147/DDDT.S86301

6. Decuypere F, Sermon J, Geerts P, et al. Treatment continuation of four long-acting antipsychotic medications in the Netherlands and Belgium: a retrospective database study. PLoS One. 2017;12(6):e0179049. doi:10.1371/journal.pone.0179049

7. Mathews M, Gopal S, Nuamah I, et al. Clinical relevance of paliperidone palmitate 3-monthly in treating schizophrenia. Neuropsychiatr Dis Treat. 2019;15:1365-1379. doi:10.2147/NDT.S197225

8. Rubio JM, Schoretsanitis G, John M, et al. Psychosis relapse during treatment with long-acting injectable antipsychotics in individuals with schizophrenia-spectrum disorders: an individual participant data meta-analysis. Lancet Psychiatr. 2020;7:749-761. doi:10.1016/S2215-0366(20) 30264-9

9. Tiihonen J, Mittendorfer-Rutz E, Majak M, et al. Real-world effectiveness of antipsychotic treatments in a nationwide cohort of 29,823 patients with schizophrenia. JAMA Psychiatr. 2017;74:686-693. doi:10.1001/jamapsychiatry.2017.1322

10. Olivares JM, Pinal B, Cinos C. Comparison of long-acting antipsychotics injection and oral antipsychotics in schizophrenia. Neuropsychiatry. 2011;1(3):275-289. doi:10.2217/npy.11.24

11. Lamb YN, Keatin GM. Paliperidone palmitate intramuscular 3-monthly formulation: a review in schizophrenia. Drugs. 2016;76:1559-1566. doi:10.1007/s40265-016-0645-5

12. Garcia-Portilla MP, Llorca PM, Maina G, et al. Symptomatic and functional outcomes after treatment with paliperidone palmitate 3-month formulation for 52 weeks in patients with clinically stable schizophrenia. Ther Adv Psychopharmacol. 2020;10:2045125320926347. doi:10.1177/ 2045125320926347

13. Finnerty MT, Layman DM, Chen Q, et al. Use of a web-based shared decision-making program: impact on ongoing treatment engagement and antipsychotic adherence. Psychiatr Serv. 2018;69(12):1215-1221. doi:10.1176/appi.ps.201800130

14. Pietrini F, Albert U, Ballerini A, et al. The modern perspective for long-acting injectables antipsychotics in the patient-centered care of schizophrenia. Neuropsychiatr Dis Treat. 2019;15:1045-1060. doi:10.2147/NDT.S199048

15. Schottle D, Schimmelmann BG, Ruppelt F, et al. Effectiveness of integrated care including therapeutic assertive community treatment in severe schizophrenia-spectrum and bipolar I disorders: four-year follow-up of the ACCESS II study. PLoS One. 2018;13(2):e0192929. doi:10.1371/ journal.pone.0192929

16. Cassidy C, Miles W. New Zealand community mental healthcare provider experience in schizophrenia management with 3-monthly paliperidone palmitate. Australas Psychiatry. 2021;29(3):299-304. doi:10.1177/1039856220928867

17. Pai N, Matthew W. Early Australian experience in the maintenance of schizophrenia management with 3-monthly paliperidone palmitate. Australas Psychiatr. 2018;26(6):628-634. doi:10.1177/1039856218789770

18. Rise MB, Stølan LO, Erdner A, et al. Patients' perspectives on three-monthly administration of antipsychotic treatment with paliperidone palmitate - a qualitative interview study. Nord J Psychiatry. 2020;75(4):257-265. doi:10.1080/08039488.2020.1841289

19. American Psychiatric Association DSM-5 Task Force. Diagnostic and Statistical Manual of Mental Disorders, 5th Edition (DSM-5). Washington, DC: American Psychiatric Association; 2013.

20. Vernon M, Revicki D, Awad G, et al. Psychometric evaluation of the Medication Satisfaction Questionnaire (MSQ) to assess satisfaction with antipsychotic medication among schizophrenia patients. Schizophr Res. 2020;118:271-278. doi:10.1016/j.schres.2010.01.021

21. Lako I, Bruggeman R, Liemburg E, et al. A brief version of the Subjects' Response to Antipsychotics questionnaire to evaluate treatment effects. Schizophr Res. 2013;147(1):175-180. doi:10.1016/j.schres.2013.02.027

22. IBM Corp. IBM SPSS Statistics for Windows, Version 25.0. Armonk, NY: IBM Corp; 2017. 
23. Fernández-Miranda JJ, Díaz-Fernández S, De berardis D, López-Muñoz F. Paliperidone palmitate every three months (PP3M) 2-year treatment compliance, effectiveness and satisfaction compared with paliperidone palmitate-monthly (PP1M) in people with severe schizophrenia. $J$ Clin Med. 2021;10(7):1408. doi:10.3390/jcm10071408

24. Patel C, El Khoury A, Huang A, Wang L, Baser O, Joshi K. Health outcomes among patients diagnosed with schizophrenia in the US veterans health administration population who transitioned from once-monthly to once-every-3-month paliperidone palmitate: an observational retrospective analysis. Adv Ther. 2019;36(10):2941-2953. doi:10.1007/s12325-019-01039-9

25. Lyer S, Banks N, Roy MA, et al. A qualitative study of experiences with and perceptions regarding long-acting injectable antipsychotics: part II-physician perspectives. Can J Psychiatry. 2013;58:23S-29S. doi:10.1177/088740341305805s04

26. Lindemayer JP, Glick ID, Talreia H, Underriner M. Persistent barriers to the use of long-acting injectable antipsychotics for the treatment of schizophrenia. J Clin Psychopharmacol. 2020;40:346-349. doi:10.1097/JCP.0000000000001225

27. Patel MX, Bent-Ennakhil N, Sapin C, et al. Attitudes of European physicians towards the use of long-acting injectable antipsychotics. BMC Psychiatr. 2020;20(1):123. doi:10.1186/s12888-020-02530-2

28. Jazieh AR, Volker S, Taher S. Involving the family in patient care: a culturally tailored communication model. Glob $J$ Qual Saf Healthc. 2018;1:33-37. doi:10.4103/JQSH.JQSH_3_18

29. National Council for Behavioral Health. Guide to long-acting medications, for providers and organizations. National Council for Behavioral Health; 2019. Available from: TheNationalCouncil.org. Accessed February 11, 2022.

30. Jaeger M, Rossler W. Attitudes towards long-acting depot antipsychotics: a survey of patients, relatives and psychiatrists. Psychiatry Res. 2010;175:58-62. doi:10.1016/j.psychres.2008.11.003

31. Parellada E, Bioque M. Barriers to the use of long-acting injectable antipsychotics in the management of schizophrenia. CNS Drugs. 2016;30 (8):689-701. doi:10.1007/s40263-016-0350-7

32. Pungor K, Sanchez P, Pappa S, et al. The Patient, Investigator, Nurse, Carer Questionnaire (PINC-Q): a cross-sectional, retrospective, non-interventional study exploring the impact of less frequent medication administration with paliperidone palmitate 3-monthly as maintenance treatment for schizophrenia. BMC Psychiatr. 2021;21(1):300. doi:10.1186/s12888-021-03305-z

33. Potkin S, Bera R, Zubek D, Lau G. Patient and prescriber perspectives on long-acting antipsychotics and analysis of in-office discussion regarding LAI treatment for schizophrenia. BMC Psychiatr. 2013;13:261. doi:10.1186/1471-244X-13-261

34. Shah A, Xie L, Kariburyo F, Zhang Q, Gore M. Treatment patterns, healthcare resource utilization and costs among schizophrenia patients treated with long-acting injectable versus oral antipsychotics. Adv Ther. 2018;35(11):1994-2014. doi:10.1007/s12325-018-0786-x

35. Pesa JA, Dosh D, Wang L, Yuce H, Baser O. Health care resource utilization and costs of California Medicaid patients with schizophrenia treated with paliperidone palmitate once monthly or atypical oral antipsychotic treatment. Curr Med Res Opin. 2017;33(4):723-731. doi:10.1080/ 03007995.2016 .1278202

36. Kane JM, Aguglia E, Altamura AC, et al. Guidelines for depot antipsychotic treatment in schizophrenia. Eur Neuropsychopharmacol. 1998;8 (1):55-66. doi:10.1016/s0924-977x(97)00045-x

37. Einarson TR, Beresa BG, Llnares IG, Moro BGM, Tedouri F, van Impe C. Cost-effectiveness of 3-month paliperidone treatment for chronic schizophrenia in Spain. J Med Econ. 2017;20(10):1039-1047. doi:10.1080/13696998.2017.1351370

38. Bouwmans C, De Jong K, Timman R, et al. Feasibility, reliability and validity of a questionnaire on healthcare consumption and productivity loss in patients with a psychiatric disorder (TiC-P). BMC Health Serv Res. 2013;13:217. doi:10.1186/1472-6963-13-217

\section{Publish your work in this journal}

Patient Preference and Adherence is an international, peer-reviewed, open access journal that focusing on the growing importance of patient preference and adherence throughout the therapeutic continuum. Patient satisfaction, acceptability, quality of life, compliance, persistence and their role in developing new therapeutic modalities and compounds to optimize clinical outcomes for existing disease states are major areas of interest for the journal. This journal has been accepted for indexing on PubMed Central. The manuscript management system is completely online and includes a very quick and fair peer-review system, which is all easy to use. Visit http://www.dovepress.com/testimonials.php to read real quotes from published authors.

Submit your manuscript here: https://www.dovepress.com/patient-preference-and-adherence-journal 